\title{
Beneficios del aula multimedia en la enseñanza del diseño para la comunicación gráfica: el caso de la Licenciatura en Diseño para la Comunicación Gráfica del Centro Universitario de Arte, Arquitectura y Diseño de la Universidad de Guadalajara
}

\author{
The Benefits of a Multimedia Classroom in Teaching Visual \\ Communication Design: The Case of the Undergraduate Degree Program \\ in Visual Communication Design in the Department of Art, Architecture \\ and Design at the University of Guadalajara
}

Eva Guadalupe Osuna Ruiz eva.osuna@cuaad.udg.mx Centro Universitario de Arte, Arquitectura y Diseño Universidad de Guadalajara Guadalajara, Jalisco, México ORCID: 0000-0001-7944-439X
Miguel Ángel Casillas López miguel.casillas@cuaad.udg.mx

Universidad de Guadalajara

Centro Universitario de Arte,

Arquitectura y Diseño

Guadalajara, Jalisco, México

ORCID: 0000-0002-5716-2970
Luis Alberto Mendoza Navarro luis.mendoza@cuaad.udg.mx Universidad de Guadalajara Centro Universitario de Arte, Arquitectura y Diseño Guadalajara, Jalisco, México ORCID: 0000-0003-2934-0191

Recibido: 21 de noviembre de 2016 Aprobado: 03 de enero de 2017 Publicado: 31 de enero de 2017

\section{Resumen:}

En el presente trabajo, se exploran los beneficios para los procesos de aprendizaje, de la incorporación de equipos para el uso de TIC en aulas de la Licenciatura en Diseño Gráfico del Centro Universitario de Arte, Arquitectura y Diseño (CUAAD) de la Universidad de Guadalajara, México. El modelo de equipamiento tecnológico estuvo compuesto por pantallas de 80 pulgadas, equipo de videoconferencia, red inalámbrica y permanencia en la nube para apoyar las actividades del profesor y del alumno. Se analiza la pertinencia del uso de estas herramientas y sus aportes a la formación académica en la mencionada licenciatura.

Se presentan las características de la remodelación e instalación de equipo tecnológico en las aulas de un centro universitario de la Universidad de Guadalajara. Actualmente, el equipamiento tecnológico en los espacios académicos se ha vuelto necesario en las instituciones educativas, pues la incorporación de tecnologías a modelos pedagógicos tradicionales permite optimizar el proceso de enseñanza aprendizaje.

Palabras clave: TIC y educación, aula multimedia, tecnología pedagógica, comunicación gráfica.
Abstract:

This paper surveys how the incorporation of educational technology tools benefits the learning process in the classrooms of the Undergraduate Degree Program in Visual Communication Design in the Department of Art, Architecture and Design at the University of Guadalajara, Mexico. The model for technological outfitting consisted of 80 inch screens, video conference equipment, Wi-Fi and cloud storage to support the activities of both professors and students. This paper analyzes the relevance of this equipment and its contribution to academic development in this undergraduate degree program.

We present the characteristics of the remodel and the installation of technology tools in classrooms in a Department of the University of Guadalajara. Technology tools have become necessary in the academic spaces of educational institutions and the incorporation of technology in traditional pedagogical models optimizes the teaching-learning process.

Keywords: ICT and education, multimedia classrooms, educational technology, visual communication. 


\section{Introducción}

Las tecnologías en el ámbito educativo
$\mathfrak{L}$ as instituciones educativas ofrecen al alumno el aprendizaje en la formación que él o ella elija. Ese aprendizaje, uno de los principales objetivos de las universidades, habrá de lograrse mediante un abanico de opciones, entre las cuales se encuentra el uso de las tecnologías de la información y la comunicación (TIC), que en la actualidad se afianza en la formación del alumnado. Tal situación obliga a replantear los planes de estudio dentro de los modelos educativos para incorporar en ellos la aplicación de estas herramientas. La Universidad de Guadalajara privilegia el desarrollo de los futuros profesionales en la sociedad. Esta función es, en parte, favorecida por la inserción de tecnologías capaces de aportar beneficios en la trayectoria académica del estudiante; de esta manera la institución cumple con el compromiso de garantizar formación especializante a su alumnado.

Para hablar de la introducción de las TIC en la educación hay que remontarse a los años ochenta, cuando los ordenadores personales se comenzaron a emplear en el sector educativo. En los años noventa las computadoras de escritorio empezaron a distribuirse en el sector laboral y en los hogares, mientras se comenzaban a incorporar a las aulas de las escuelas, equipos de cómputo; estos espacios se denominaron laboratorios de cómputo o de informática. Este fenómeno propició la readaptación de los planes curriculares académicos para la enseñanza de esta nueva disciplina. Al aparecer internet el impacto fue aún mayor, ya que la red de redes facilitó el acceso a la información, así como el trabajo colaborativo en línea. Comenzó entonces el uso intensivo de herramientas de comunicación como el correo electrónico, los chats, los foros y los cursos en línea (Hosy, 2013, pág. 81).

Las instituciones educativas apostaron por implementar las TIC en sus planes académicos, integrando a la docencia la difusión y la generación de conocimientos en el uso de estas tecnologías (Merril et al., 1996, citado en Hosy, 2013). El proceso de integración consiste en "combinar las TIC y procedimientos de enseñanza tradicional para producir aprendizaje” (pág. 82).

En los inicios de la incorporación de las TIC en la educación superior no se esperaba las aportaciones que se han dado en el proceso de enseñanza-aprendizaje. 
Según Riascos, Quintero y Ávila (2009) "uno de los factores principales para el éxito de la implementación de las TIC en la educación superior es la percepción de los docentes frente a ellas" (pág. 134).

Las tecnologías para la información y comunicación promueven nuevos ambientes de aprendizaje. Hooper y Rieber (1995), citados por Jaramillo, Castañeda y Pimienta (2009) identifican algunas etapas en el uso de las TIC para apoyar el aprendizaje: familiarización, utilización, integración, reorientación, evolución, transmisión de mensajes, y facilidad en la interacción; concluyen que el docente es quien incorpora, integra y usa las herramientas tecnológicas.

Otro de los actores primordiales en la integración de las TIC en la educación es el alumno. Consideramos que, más que un participante del proceso, es realmente el objetivo-meta, a quien van orientadas todas las estrategias que puedan abonar a su formación.

La interactividad profesor-alumno y alumno-alumno y, desde nuestra perspectiva, profesor-alumno-TIC, conducen hacia un proceso compartido, en el cual se encuentran los significados y los conocimientos que finalmente abonan a esta construcción del aprendizaje (Baelo, 2008).

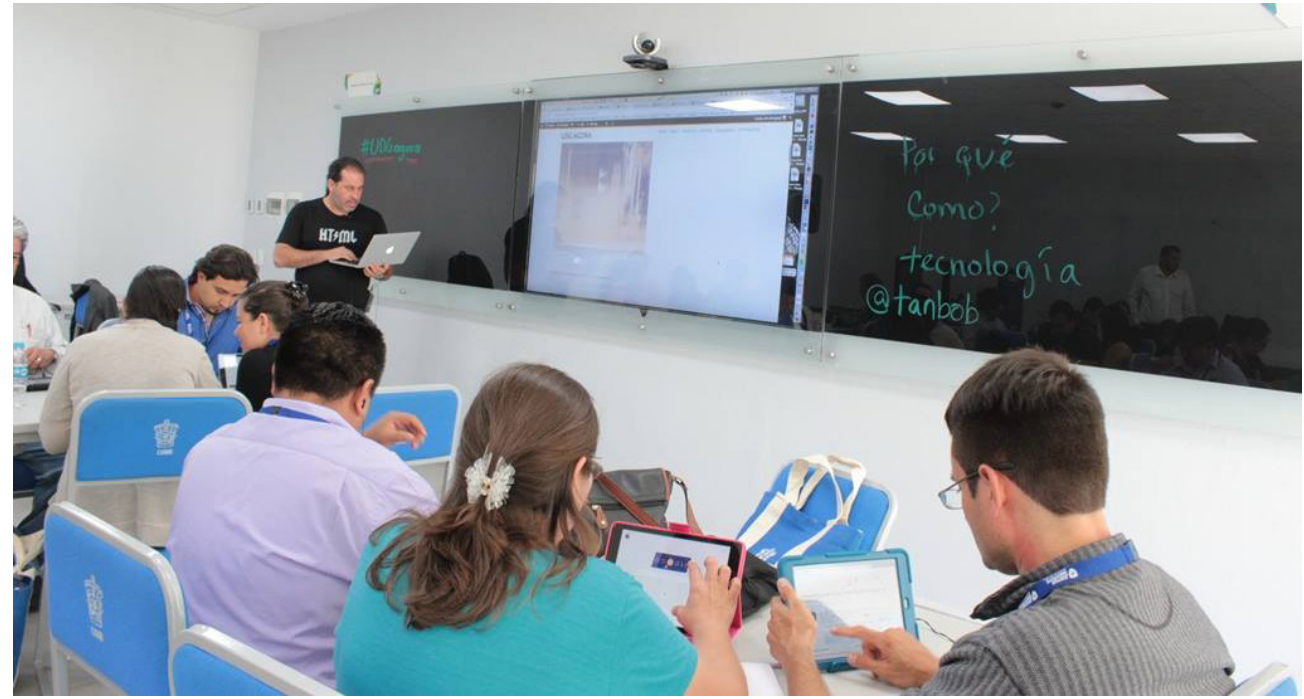

Figura 1. Profesor y estudiantes colaborando en un aula multimedia. Fuente: Coordinación de Tecnologías para el Aprendizaje, 2016.

Para finalizar este apartado, Bates (2001, págs. 48-49, en López de la Madrid, 2007) menciona algunas ventajas de incluir las TIC en la enseñanza, entre las cuales se encuentran: la enseñanza y aprendizaje disponible en todo momento, el fácil acceso a la información, la mejora de la comunicación profesor-alumno y el refuerzo de contenidos referentes al tema con profesores de cualquier parte del mundo gracias a la ruptura de barreras de la distancia mediante las videoconferencias, aplicadas como herramientas para el proceso de enseñanza-aprendizaje. 
La introducción de TIC en el ámbito educativo implica que además de su inclusión en los espacios educativos, se les dé uso a esos espacios, siendo esta última acción tan importante como la primera, dado que si los recursos existen en el aula pero no se usan, la inversión para adquirirlos no tendrá el efecto esperado en los modelos educativos de la institución.

Las aulas multimedia en la Licenciatura en Diseño para la Comunicación Gráfica
La Universidad de Guadalajara, la universidad pública del estado de Jalisco, está constituida por centros universitarios regionales y temáticos. Los primeros se encuentran fuera de la zona metropolitana de Guadalajara y tienen diversas licenciaturas, de acuerdo con de las necesidades de cada región, mientras que los segundos se encuentran en la zona metropolitana de Guadalajara y agrupan licenciaturas de áreas disciplinares semejantes. Este es el caso del Centro Universitario de Arte, Arquitectura y Diseño (CUAAD) que alberga nueve licenciaturas, entre las cuales se encuentra la Licenciatura en Diseño para la Comunicación Gráfica (LDCG).

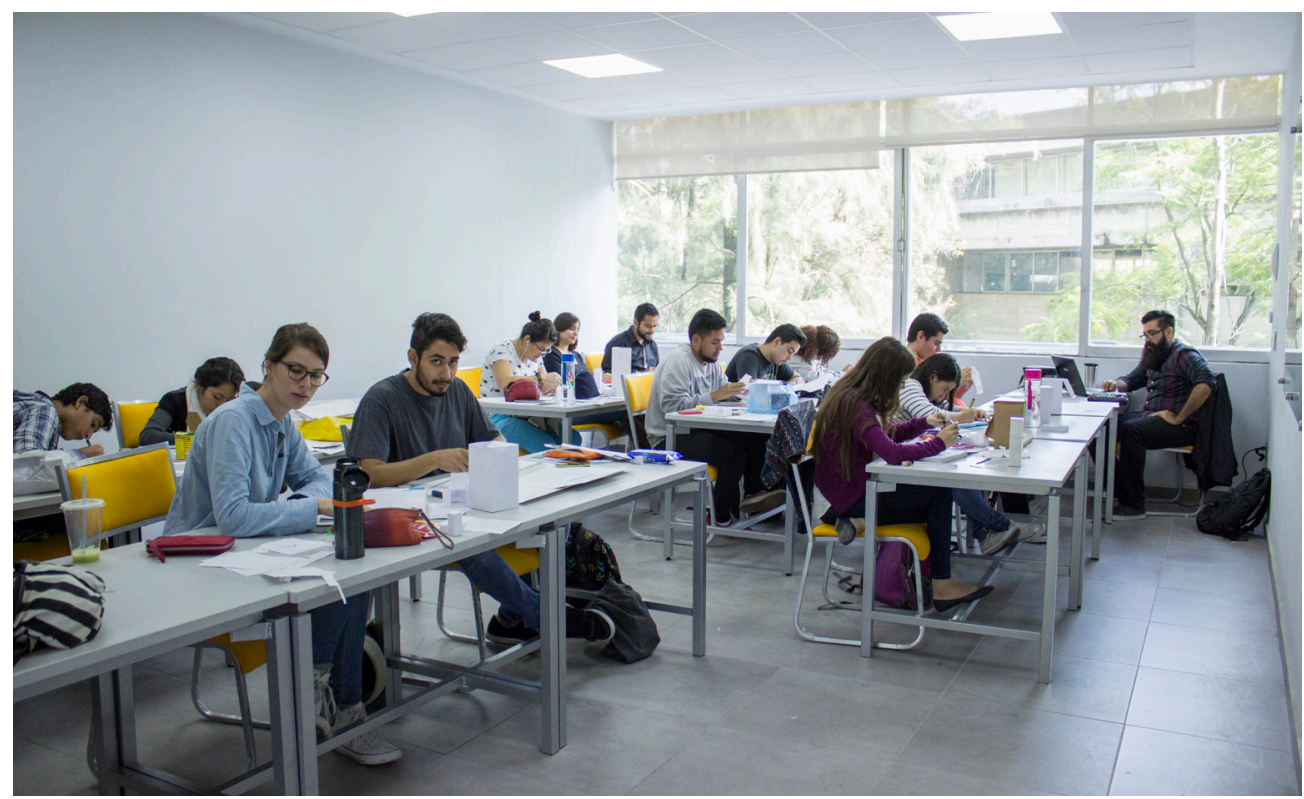

Figura 2. Alumnos de la materia de Diseño VII de la Licenciatura en Diseño para la

Comunicación Gráfica. Fuente: Coordinación de Tecnologías para el Aprendizaje, 2016.

Es necesario conocer un poco sobre la LDCG para comprender su desarrollo y la manera en que impacta la implementación de las aulas multimedia en el desarrollo de las clases. De acuerdo con la página de internet del CUAAD ("Oferta académica," s.f.):

La licenciatura en Diseño y Comunicación Gráfica fue creada y aprobada a partir del 1 de septiembre de 1990, posteriormente en el año 1994 se propone la modificación al plan de estudios de la Licenciatura en Diseño para la Comunicación Gráfica. En el año 1996 el plan de estudios de la Licenciatura se rige por el sistema de créditos, en el cual se establecen áreas determinadas 
con un valor de créditos de cada materia y un valor global en la base de los requisitos establecidos de cada área, quedando 4 áreas de formación:

Básica común obligatoria

Básica particular obligatoria

Especializante obligatoria

Optativa abierta

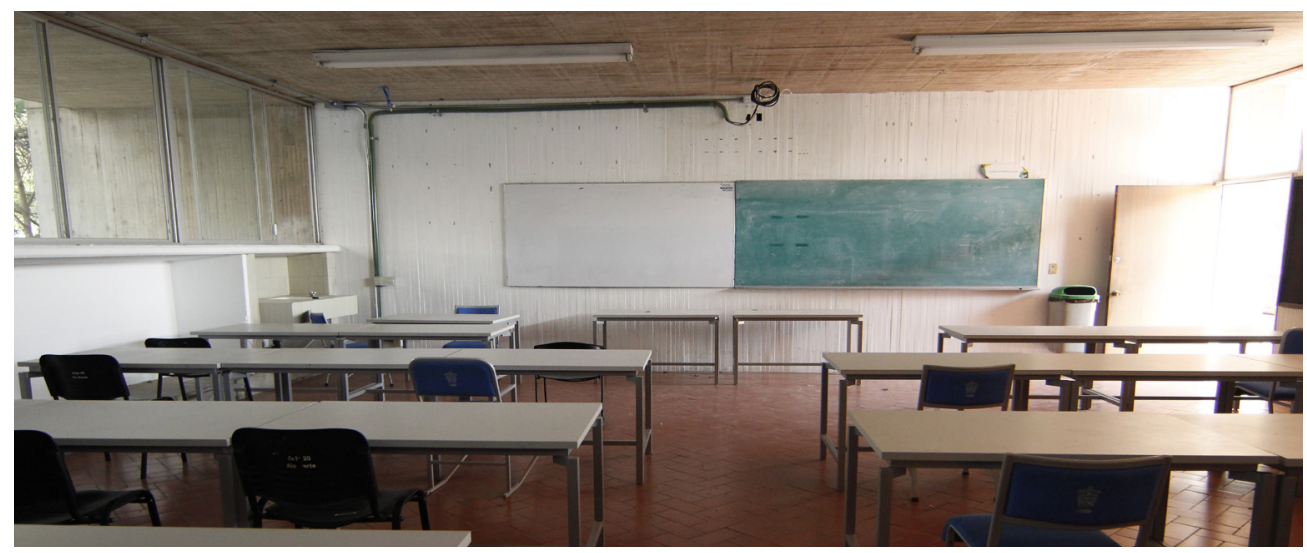

Figura 3. Ejemplo de aula antes de la intervención.

Fuente: Coordinación de Tecnologías para el Aprendizaje, 2016.

En las áreas de formación básica común obligatoria predominan las asignaturas que son teóricas y teórico-prácticas; en el área particular obligatoria, las teórico-prácticas; y tanto el área especializante como la de asignaturas optativas se enfocan en las asignaturas prácticas, por lo tanto, las aulas tienen características apropiadas para estos tipos de asignaturas. Antes de 2014 estos espacios sólo contaban con mobiliario básico (mesas, bancos y pizarrón).

A partir de 2014 el CUAAD, en atención al cumplimiento de sus funciones sustantivas y adjetivas como institución de educación superior, gestionó una infraestructura tecnológica robusta que respondiera a las necesidades de su comunidad académica. En este tenor el centro universitario realizó renovaciones orientadas a la infraestructura tecnológica, como fortalecer el servicio de red e implementar laboratorios y equipo de cómputo, entre otras.

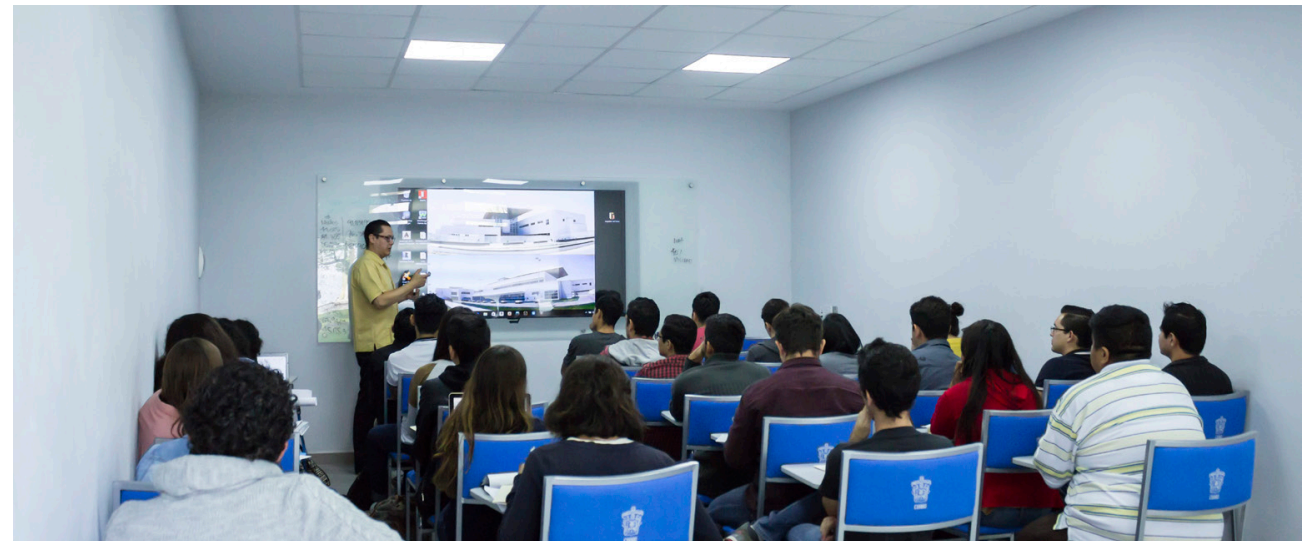

Figura 4. Ejemplo de aula para asignaturas teóricas. Fuente: Coordinación de Tecnologías para el Aprendizaje, 2016. 
El CUAAD tenía cerca de treinta aulas en 2014 , que contaban con proyector y pantalla de proyección; en los espacios desprovistos de estos equipos el profesor solicitaba su préstamo a la Coordinación de Tecnologías o al departamento de adscripción, pero en ocasiones eran insuficientes para cubrir la demanda.

En el proyecto de infraestructura tecnológica el objetivo fue solucionar esta problemática, además de implementar nuevas herramientas, que al integrarse en las clases apoyaran en la mejora del proceso de enseñanza-aprendizaje, tomando en cuenta también una mejora en la infraestructura física.

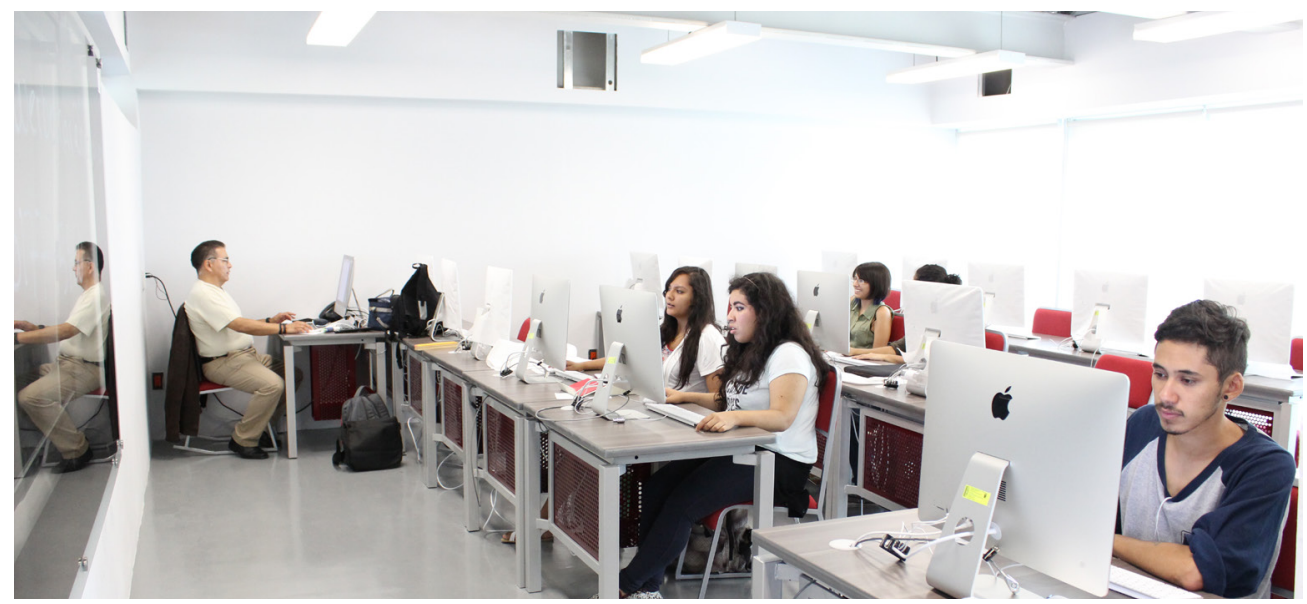

Figura 5. Ejemplo de aula para asignaturas teórico-prácticas. Fuente: Coordinación de Tecnologías para el Aprendizaje, 2016.

En este sentido, en las aulas se realizó la instalación de equipo tecnológico, transformando estos espacios en aulas multimedia, con la finalidad de apoyar las estrategias pedagógicas en el proceso de aprendizaje del estudiante.

Las pantallas adquiridas cuentan con puertos de transmisión de audio y video de alta definición en dimensiones de 80 y 75 pulgadas. Están equipadas con interfaces de conexión de video VGA y HDMI con audio de alta definición, además de un puerto RS232 para control y mini plug para audio.

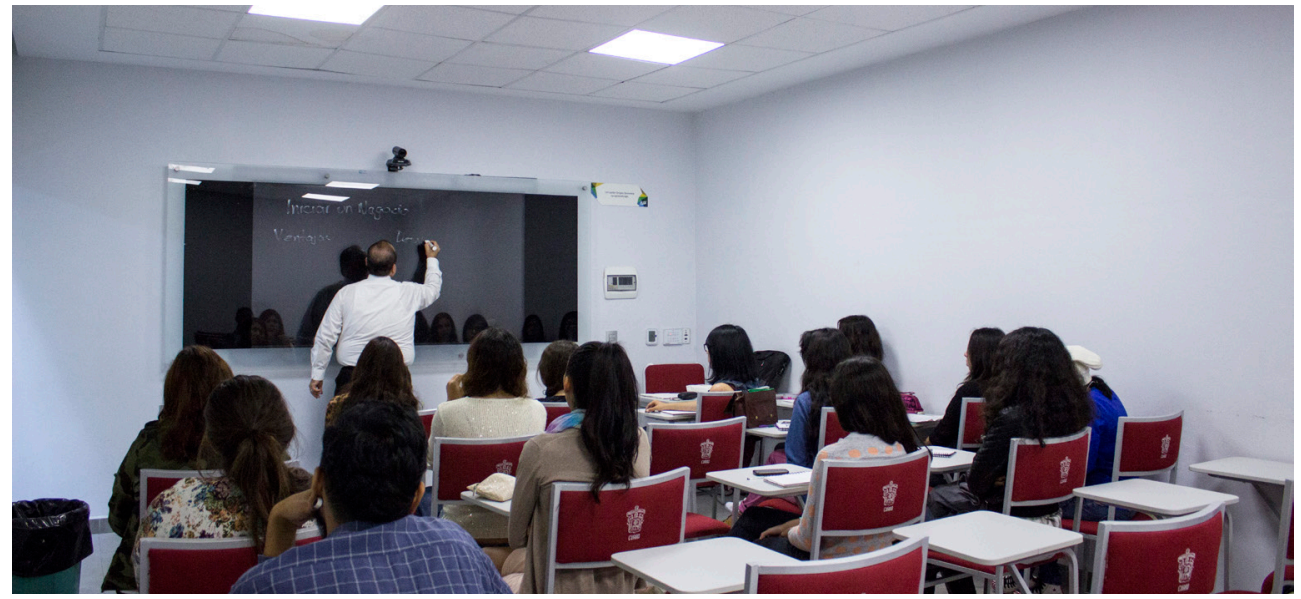

Figura 6. Pantalla en aula multimedia, con soporte de vidrio para escribir, en lugar de pintarrón o pizarrón. Fuente: Coordinación de Tecnologías para el Aprendizaje, 2016. 


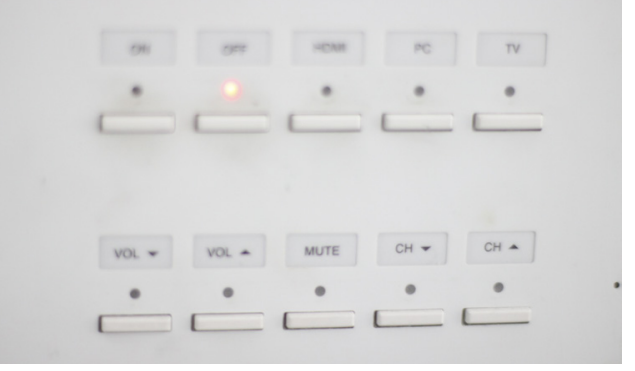

Figura 7. Botonera para la interconexión con la pantalla. Fuente: Coordinación de Tecnologías para el Aprendizaje, 2016.
A través de una placa de control con 10 botones programables instalada ergonómicamente en el espacio del profesor se enciende y apaga la pantalla, se controla el volumen y la selección de entrada de video, con funciones reservadas para futuros proyectos. Además, se cuenta con una placa de conexiones, en donde mediante cables VGA o HDMI se conectan los equipos de cómputo y otros dispositivos electrónicos.

Desde la aparición de la internet los sectores de la sociedad han cambiado sus procedimientos, en algunos casos de manera total, lo que ha derivado en mejores resultados. Dentro del sector educativo uno de los cambios significativos ha sido la interacción en red, lo que ha facilitado la transmisión de conocimientos a partir del e-learning.

En algunas de las aulas se realizó la instalación de un equipo de videoconferencia con la finalidad de celebrar sesiones entre aulas, entre campus de la Universidad de Guadalajara o hasta cualquier parte del mundo.

Con esta implementación se acercan a los alumnos ponencias en cualquier momento, sin necesidad de trasladar al conferencista al centro universitario. Otra ventaja es que el docente provee servicio en línea a los alumnos que por alguna circunstancia estén ausentes.

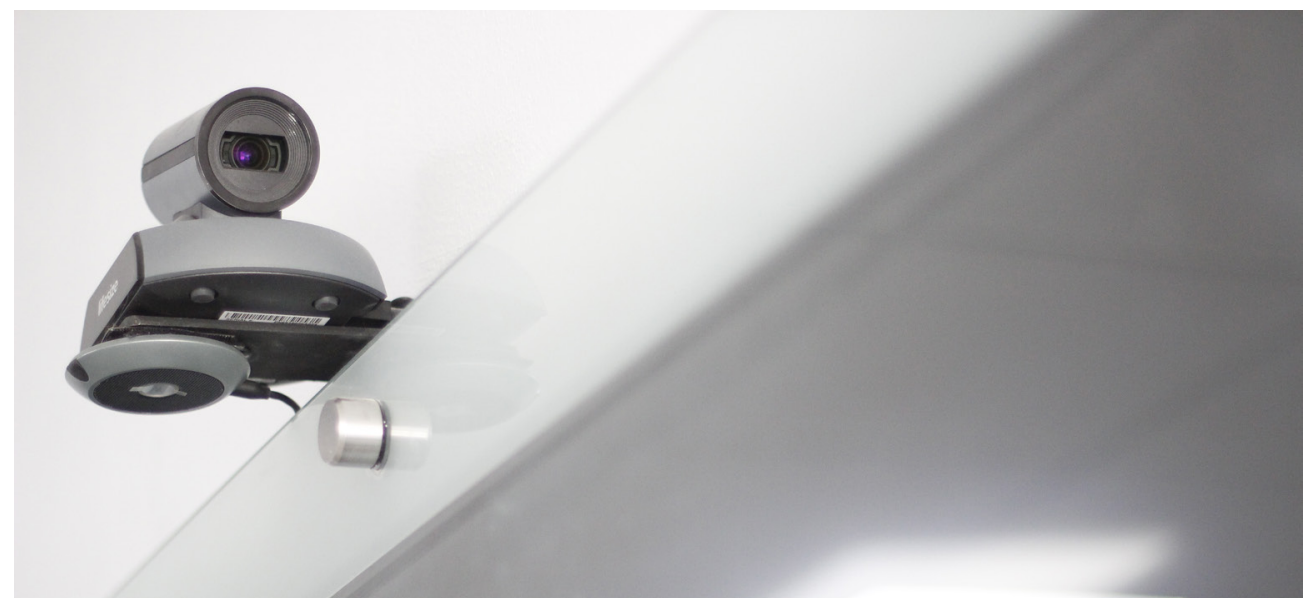

Figura 8. Equipo para la realización de videoconferencias. Fuente: Coordinación de Tecnologías para el Aprendizaje, 2016.

Con la incursión de la internet las TIC cobraron mayor fuerza, se abrió una extensa gama de actividades derivadas de la conjugación de herramientas que permitieron a los usuarios conectarse desde el aula de clases. Con ello se tenía ya la posibilidad de utilizar los recursos que brinda la red de redes, pues anteriormente la señal de internet no llegaba a todos los salones a causa de los muros de concreto con que está construido el centro universitario; solamente se había reforzado 
la señal en el ciberjardín del campus. Actualmente el proyecto de red inalámbrica contempla contar con un equipo de acceso a internet por cada aula, para brindar este servicio a todos los dispositivos que se encuentren dentro del campus.

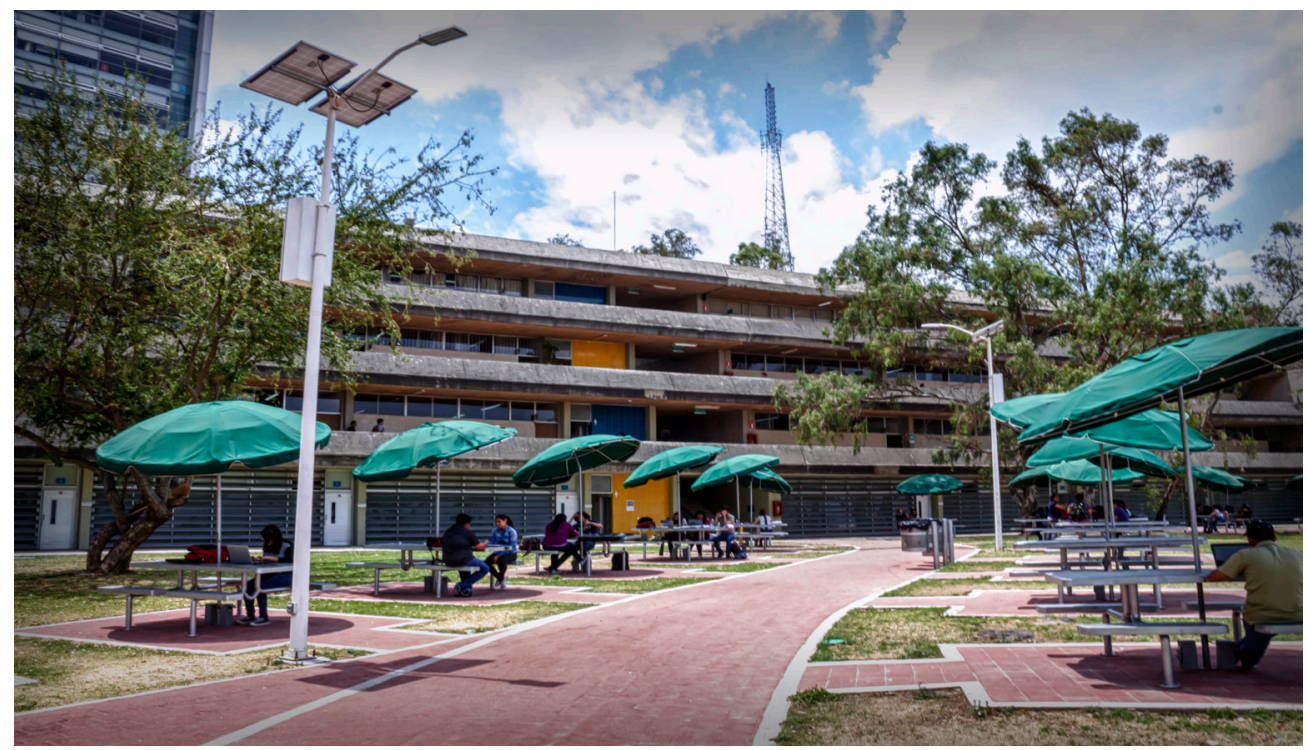

Figura 9. Ciberjardín en donde los estudiantes pueden trabajar con acceso a internet. Fuente: Coordinación de Tecnologías para el Aprendizaje, 2016.

Como puede observarse, son muchos los recursos tecnológicos que se han implementado, siguiendo las directrices de la administración para la mejora del proceso de enseñanza-aprendizaje.

Impacto del aula multimedia
Aunque la implementación de las aulas es para todas las licenciaturas que alberga el CUAAD, solamente se presentan los resultados obtenidos en la Licenciatura en Diseño para la Comunicación Gráfica, puesto que las asignaturas de diseño son el eje principal de la carrera. En ellas convergen las competencias desarrolladas por los estudiantes en el resto de las asignaturas, tales como Fundamentos del diseño, Fotografía, Video, Computación y Expresión gráfica, entre otras. La carrera contempla siete niveles de diseño, en los cuales se aplicaron encuestas para el levantamiento de datos acerca de los beneficios obtenidos con la implementación de las aulas multimedia; esto, con la finalidad de identificar la visión de los estudiantes y de los profesores respecto a los recursos tecnológicos instalados en las mismas.

Los resultados de la encuesta realizada en el ciclo escolar 2016-B a 46\% de los docentes que integran la plantilla académica de la LDCG permitieron trazar un panorama de la mejora en el proceso de enseñanza-aprendizaje, a la vez que dan luz sobre qué se debe hacer para mejorar el servicio y prevenir problemas que el uso de la tecnología podría causar. 
Se preguntó a los docentes acerca del nivel de frecuencia con que usan los recursos tecnológicos de las aulas multimedia (internet, equipo de videoconferencia y pantalla). Con respecto al promedio de su uso (figura 10) el servicio de internet es uno de los más utilizados; este servicio se provee mediante equipos de red inalámbrica y su acceso se realiza mediante el código de profesor o estudiante. La pantalla le sigue en frecuencia de uso. Así mismo, se observa que pocos profesores utilizan el equipo de videoconferencia.

INDICA EL PORCETANJE DE LA FRECUENCIA CON QUE TU PROFE USA LOS RECURSOS TECNOLÓGICOS DE LAS AULAS MULTIMEDIA EN SUS CLASES

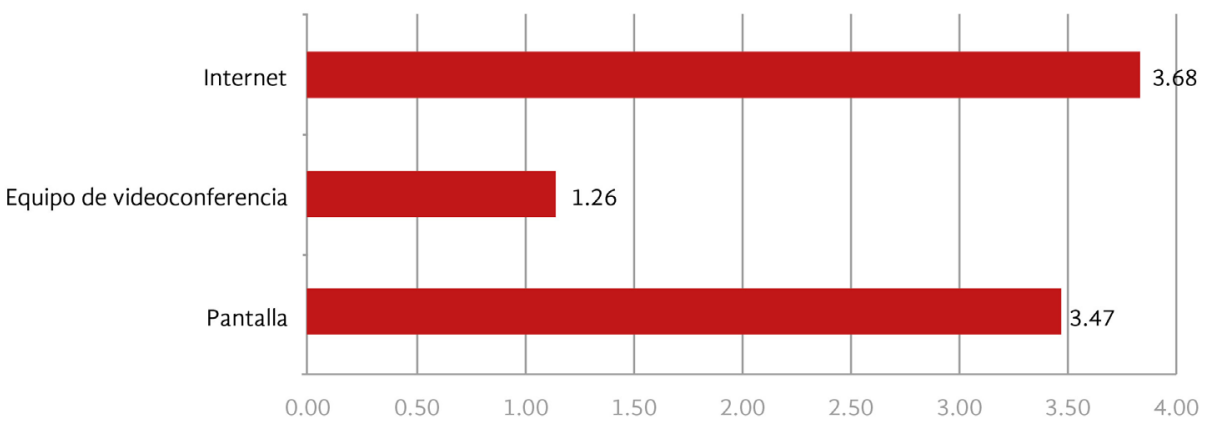

Figura 10. Promedio de la frecuencia con que los docentes utilizan el equipo multimedia en el aula. Fuente: encuesta aplicada.

Con respecto a los porcentajes de los docentes que respondieron sobre la frecuencia con que utilizaban los recursos tecnológicos durante el semestre, destaca que $63.16 \%$ de los encuestados usan de tres cuartas partes del semestre a todo el semestre la pantalla; $73.68 \%$ de los encuestados utiliza en un mayor porcentaje el internet en sus clases, datos que contrastan con el $78.95 \%$ de docentes que no utilizan el equipo de videoconferencia.

\begin{tabular}{|c|c|c|c|c|}
\hline & $0 \%-25 \%$ & $26 \%-50 \%$ & $51 \%-75 \%$ & $76 \%-100 \%$ \\
\hline Pantalla & $5.26 \%$ & $5.26 \%$ & $26.32 \%$ & $63.16 \%$ \\
\hline Equipo de videoconferencia & $78.95 \%$ & $15.79 \%$ & $5.26 \%$ & $0 \%$ \\
\hline Red inalámbrica & $0 \%$ & $5.26 \%$ & $21.05 \%$ & $73.78 \%$ \\
\hline
\end{tabular}

Tabla 1. Rango de frecuencia especificada por los profesores acerca del uso de recursos multimedia durante el semestre. Fuente: Encuesta aplicada.

Fue interesante conocer la manera en que los profesores utilizaron el servicio de internet para la enseñanza del diseño; en la tabla 2 se evidencia que un alto porcentaje de los encuestados siempre lo hace para buscar información y compartir archivos, seguido por la presentación de videos. En un porcentaje menor en todos los niveles de frecuencia se encuentra el trabajo simultáneo en archivos.

\begin{tabular}{|l|c|c|c|c|}
\hline & SIEMPRE & MUCHO & PoCO & NUNCA \\
\hline Búsqueda de información & $42.11 \%$ & $57.89 \%$ & $0 \%$ & $0 \%$ \\
\hline Presentar videos & $31.58 \%$ & $42.11 \%$ & $26.32 \%$ & $0 \%$ \\
\hline Compartir archivos (drive, dropbox) & $42.11 \%$ & $31.58 \%$ & $26.32 \%$ & $0 \%$ \\
\hline Trabajo simultaneo en archivos (drive) & $21.05 \%$ & $36.84 \%$ & $26.32 \%$ & $16 \%$ \\
\hline
\end{tabular}

Tabla 2. Rango de frecuencia especificada por los profesores acerca del uso de recursos en línea durante el semestre. Fuente: Encuesta aplicada. 
Otros usos que declararon los docentes: trabajo en equipos con uso de Pinterest, dinámicas con la herramienta Kahoot!, producción de contenido para blogs educativos, referencias académicas en relación directa con la clase, aulas virtuales y el uso de la plataforma Schoology.

En la tabla 3 se muestra la mejora que los docentes han percibido a partir de la implementación de las aulas multimedia, dado que ha mejorado la optimización del tiempo, la innovación en el material que usan para la clase y la implementación de estrategias didácticas más dinámicas. En segundo lugar, pero con un alto porcentaje de respuestas, se considera que ha mejorado mucho la atención de los estudiantes y por consiguiente el proceso de enseñanza-aprendizaje. En tercer lugar, $78.95 \%$ valora el aumento de la generación de un ambiente colaborativo.

\begin{tabular}{|l|c|c|c|}
\hline \multicolumn{2}{|c|}{ MucHo } & Poco & NADA \\
\hline Optimización del tiempo & $94.74 \%$ & $5.26 \%$ & $0 \%$ \\
\hline Mayor atención de los estudiantes & $89.47 \%$ & $10.53 \%$ & $0 \%$ \\
\hline Mejora en el proceso de enseñanza aprendizaje & $89.47 \%$ & $10.53 \%$ & $0 \%$ \\
\hline Favorece la memorización del concepto & $57.89 \%$ & $36.84 \%$ & $5.26 \%$ \\
\hline Fomenta la implementación de estratégias didácticas más dinámicas & $94.74 \%$ & $5.26 \%$ & $0 \%$ \\
\hline Crea un ambiente de aprendizaje colaborativo & $78.95 \%$ & $21.05 \%$ & $0 \%$ \\
\hline Innovación del material que usa el profesor para su clase & $9474 \%$ & $5.26 \%$ & $0 \%$
\end{tabular}

Tabla 3. Percepción de los docentes con respecto al nivel de mejora de las acciones mencionadas, enfocada a los beneficios del proceso de enseñanza-aprendizaje de sus alumnos. Fuente: Encuesta aplicada.

Como un punto no muy positivo se comenta la posibilidad de que el alumno se distraiga de alguna manera, dato que se contrapone con la opinión de que el uso de TIC favorece las actividades extra clase. A continuación, algunos de los comentarios de los docentes:

"Un apoyo indispensable".

"Podremos aprovechar aún más con las nuevas herramientas Google UdeG".

"Se ha incrementado la posibilidad de ofrecer material didáctico actualizado".

"Es un gran avance el tener estos recursos pues nos permiten estar a la par de otras universidades de prestigio".

"No se tiene el impacto deseado ya que en algunas aulas no hay un mantenimiento continuo a cosas sencillas como conexión a internet, conexiones en cableado y personal capacitado para asesoría".

La población de la comunidad estudiantil de la LDCG se compone de aproximadamente mil 200 alumnos, de los cuales 500 se encuentran estudiando en semestres avanzados de la licenciatura; de ellos $10 \%$ integran la muestra a la que se aplicó la encuesta. Para la selección de la muestra se tomó como criterio que los estudiantes hubieran realizado actividades de aprendizaje en las aulas remodeladas y equipadas. Los resultados fueron los siguientes: 
INDICA EL PORCETANJE DE LA FRECUENCIA CON QUE TU PROFE USA LOS RECURSOS TECNOLÓGICOS DE LAS AULAS MULTIMEDIA EN SUS CLASES

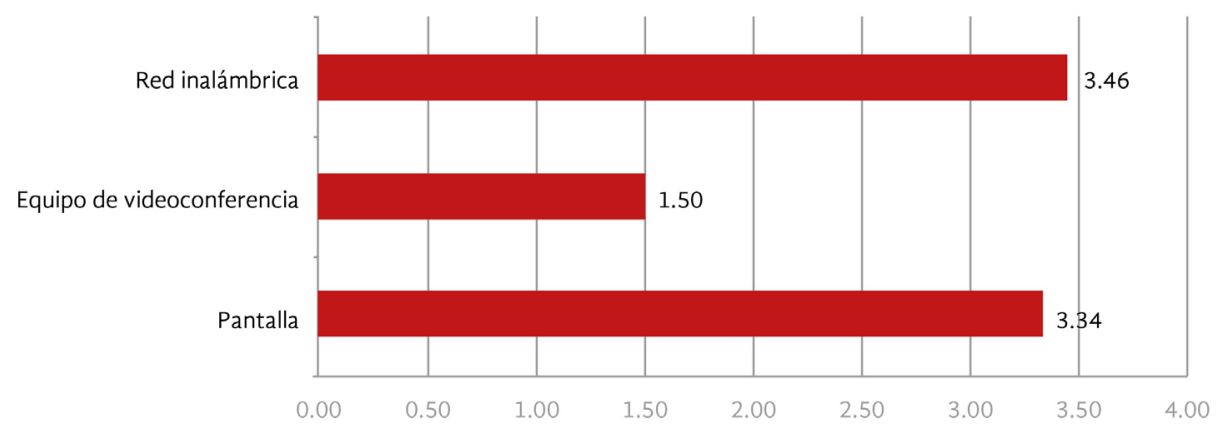

Figura 11. Promedio de la frecuencia con que los docentes utilizan el equipo multimedia en el aula, según la percepción de los estudiantes. Fuente: Encuesta aplicada.

Con respecto la frecuencia, la red inalámbrica y la pantalla es lo que más utilizan los profesores, según los estudiantes. Comparando con los resultados arrojados por los docentes, la red inalámbrica presenta $0.22 \%$ menos y el uso de la pantalla, $0.13 \%$ en el mismo rubro.

\begin{tabular}{|l|c|c|c|c|}
\hline & $\mathbf{0} \% \mathbf{- 2 5} \%$ & $\mathbf{2 6} \% \mathbf{- 5 0} \%$ & $\mathbf{5 1} \% \mathbf{- 7 5} \%$ & $\mathbf{7 6} \%-\mathbf{1 0 0} \%$ \\
\hline Pantalla & $6.90 \%$ & $13.79 \%$ & $17.24 \%$ & $62.07 \%$ \\
\hline Equipo de videoconferencia & $71.43 \%$ & $7.14 \%$ & $21.43 \%$ & $0 \%$ \\
\hline Red inalámbrica & $3.57 \%$ & $7.14 \%$ & $28.57 \%$ & $60.71 \%$
\end{tabular}

Tabla 4. Percepción de los estudiantes con respecto al porcentaje de uso de los recursos tecnológicos por parte de los docentes durante el semestre. Fuente: encuesta aplicada.

Acerca de la pantalla en el aula, los estudiantes perciben un menor uso con respecto a lo que los docentes declararon, si bien la diferencia se podría considerar insignificante; en el uso de la red inalámbrica la diferencia es un poco mayor: llega hasta una diferencia de $13 \%$; en cuanto al equipo de videoconferencia, profesores y estudiantes coinciden en que casi no se utiliza.

En la tabla 5 se puede notar congruencia con los resultados de los docentes, exceptuando la presentación de videos, que queda con una diferencia de $24.68 \%$ en la frecuencia de siempre; en los comentarios solamente se agregó la descarga de material multimedia.

\begin{tabular}{l|c|c|c|c}
\hline & SIEMPRE & MUCHO & POCO & NUNCA \\
\hline Búsqueda de información & $48.28 \%$ & $41.38 \%$ & $6.90 \%$ & $0 \%$ \\
\hline Presentar videos & $6.90 \%$ & $31.03 \%$ & $48.28 \%$ & $10.34 \%$ \\
\hline Compartir archivos (drive, dropbox) & $65.52 \%$ & $31.03 \%$ & $3.45 \%$ & $0 \%$ \\
\hline Trabajo simultaneo en archivos (drive) & $24.14 \%$ & $34.48 \%$ & $37.93 \%$ & $3.45 \%$
\end{tabular}

Tabla 5. Frecuencia con que los estudiantes realizan las acciones señaladas en el aula, durante sus clases. Fuente: encuesta aplicada.

En la tabla 6 se presenta la percepción de los estudiantes con respecto al nivel de mejora de ciertas actividades en clase. Los puntos de coincidencia con los 
docentes son la implementación de estrategias didácticas más dinámicas, la mejora en el proceso de enseñanza-aprendizaje y la generación de un ambiente de aprendizaje colaborativo. El punto en el que los estudiantes otorgaron un mayor porcentaje es la memorización de conceptos, situación que sorprende con respecto a la percepción de los profesores, pues desde la óptica de quienes están aprendiendo el uso de recursos permite mejorar este aspecto.

\begin{tabular}{|l|c|c|c}
\hline & MUCHO & Poco & NADA \\
\hline Optimización del tiempo & $72.41 \%$ & $27.59 \%$ & $0 \%$ \\
\hline Mayor atención de los estudiantes & $65.52 \%$ & $34.48 \%$ & $0 \%$ \\
\hline Mejora en el proceso de enseñanza aprendizaje & $79.31 \%$ & $20.69 \%$ & $0 \%$ \\
\hline Favorece la memorización del concepto & $65.52 \%$ & $34.48 \%$ & $0 \%$ \\
\hline Fomenta la implementación de estratégias didácticas más dinámicas & $89.66 \%$ & $10.34 \%$ & $0 \%$ \\
\hline Crea un ambiente de aprendizaje colaborativo & $79.31 \%$ & $20.69 \%$ & $0 \%$ \\
\hline Innovación del material que usa el profesor para su clase & $75.86 \%$ & $24.14 \%$ & $0 \%$
\end{tabular}

Tabla 6. Percepción de los estudiantes con respecto al nivel de mejora de las acciones mencionadas, enfocada a los beneficios del proceso de enseñanza-aprendizaje. Fuente: encuesta aplicada.

Los aspectos que resultaron en empeoramiento, de acuerdo con los estudiantes, son la optimización del tiempo (con una diferencia de $22.33 \%$ ), así como una mayor atención de los estudiantes (con una diferencia de 23.95\%) y la innovación en el material que usa el docente para la clase (diferencia de 18.88\%), situación que contrasta con el esfuerzo que el docente cree realizar.

Finalmente, los comentarios que hicieron los estudiantes fueron:

"El equipo es adecuado, han mejorado bastante, pero se necesita más educación en todos los sentidos y ambas partes (estudiantes y maestros) para que estas herramientas permanezcan en buen estado".

"Hay algunas aulas en donde sus pantallas no sirven".

"Están geniales".

"Les falta trabajo aún, en ocasiones la red no aguanta que muchos alumnos estén. conectados al mismo tiempo, pero hasta el momento se han convertido en un apoyo importante para el desarrollo del alumnado".

"Fue muy bueno que cambiaran, eso de seguir utilizando proyector ya es pasado, ahora hay más opciones al momento de presentar proyectos".

"Han mejorado muchísimo desde que entré al CUAAD, no le pide nada a las escuelas de paga".

"Me agradan, falta que las usen más".

La percepción de los estudiantes coincide con el cuidado y mantenimiento que se debe dar al equipo, y según la encuesta están de acuerdo en que se trata de una acción positiva. 
Conclusiones La implementación de las aulas multimedia ha producido muchos beneficios, entre ellos la mejora del proceso de enseñanza-aprendizaje, situación que permite que el docente prepare un material de apoyo innovador para sus clases y que use recursos libres que ayudan a dinamizar las clases.

Desde la perspectiva de los estudiantes es necesario que se utilice y aproveche más el equipo, lo cual coincide con la solicitud de los docentes para que exista una capacitación para su uso. Tanto estudiantes como docentes coincidieron en la necesidad de dar mantenimiento a los equipos, puesto que no funcionan al cien por ciento.

La implementación de TIC dentro de los espacios académicos mediante proyectos transversales de infraestructura física y tecnológica es una solución viable, además de dotar de movilidad a los usuarios mediante el uso de distintos dispositivos de videoconferencia. Los resultados señalaron la importancia de establecer lineamientos y capacitaciones para el uso de esta tecnología en los procesos de enseñanza-aprendizaje.

Pensar en implementar una solución en el uso de dispositivos móviles en las aulas, donde alumnos y profesores utilicen esta herramienta, al inicio de este proyecto era distante, pero ahora se cuenta con la infraestructura tecnológica suficiente para utilizar estas herramientas; un celular o una tableta pueden aprovecharse para realizar actividades de aprendizaje en estos espacios.

En el trabajo se presentó la solución de remodelación y equipamiento con características particulares y acordes al centro universitario. Se trata de una solución innovadora en la que el uso de pantallas presenta un reto desde la isóptica y acústica, su conectividad al usuario, su distribución dentro del aula, la implementación de equipos de videoconferencia, además de la automatización y el uso de nuevas herramientas piloto para el trabajo colaborativo.

La consolidación a largo plazo de los proyectos tecnológicos, sin duda, se centra en el soporte y el mantenimiento de los dispositivos, así como en consolidar los esfuerzos para que los distintos grupos de trabajo de la Coordinación de Tecnologías para el Aprendizaje establezcan líneas de trabajo enfocadas en mantener esta solución tecnológica.(ㄱ fica de Educomunicación. XVIII (35), 159-160. Disponible en http://ehis.ebscohost. com/ehost/pdfviewer/pdfviewer?vid=3ysid=de522c89-ea95-4604-906c-59ffa$2 \mathrm{~d} 31 \mathrm{c} 7 \mathrm{~d} \% 40$ sessionmgr4yhid $=17$

"Oferta académica". (s.f.). Portal del Centro Universitario de Arte, Arquitectura y Diseño de la Universidad de Guadalajara Recuperado el 9 de noviembre de 2016 de http://www.cuaad.udg.mx/?q=oferta/licenciaturas/ldcg 
Hosy, M. (2013) El desafío de la tecnología en las humanidades. Claves para una integración equilibrada de los usos de las TIC en el proceso de enseñanza-aprendizaje págs. 76, 81-83, 97-98, 102. Disponible en http://ehis.ebscohost.com/ehost/pdfviewer/ pdfviewer?vid=9ysid=0801fbe4-4f02-419e-8528-132b028531dd\%40sessionmgr198yhid $=102$

Jaramillo, P., Castañeda, P., y Pimienta, M. (2009) Qué hacer con la tecnología en el aula: inventario de usos de las TIC para aprender y enseñar. Educación y Educadores, 12 (2), 159-179. Disponible en http://ehis.ebscohost.com/ehost/pdfviewer/ pdfviewer?vid=9ysid=0801fbe4-4f02-419e-8528-132b028531dd\%40sessionmgr198yhid $=102$

López de la Madrid, M. (2007) Uso de las TIC en la educación superior de México. Un estudio de caso. Apertura: Revista de Innovación Educativa, 7 (7), 63-81. Disponible en http://ehis.ebscohost.com/ehost/pdfviewer/pdfviewer?vid=12ysid=0801fbe44f02-419e-8528-132b028531dd\%40sessionmgr198yhid=102

Riascos, S., Quintero, D., y Ávila, G. (2009) Las TIC en el aula: percepciones de los profesores universitarios. Educación y Educadores, 12 (3), 133-157. Disponible en http://ehis.ebscohost.com/ehost/pdfviewer/pdfviewer?vid=15ysid=0801fbe44f02-419e-8528-132b028531dd\%40sessionmgr198yhid=102

\section{Sobre los autores Mtra. Eva Guadalupe Osuna Ruiz}

Profesora de tiempo completo de la Universidad de Guadalajara (UdeG), Doctorante en Gestión de la Educación Superior y Maestra en Planeación de la Educación Superior. Imparte las materias de Metodología y Tesis I a estudiantes de la Licenciatura en Diseño para la Comunicación Gráfica; y la materia de Proyectos Artísticos I, a estudiantes de las Licenciaturas en Artes Visuales para la Expresión Plástica y Expresión Fotográfica y de las Licenciaturas en Artes Escénicas para la Expresión Teatral y Dancística. Tiene perfil PRODEP y es miembro del Cuerpo Académico: UDG-CA-790 "Procesos de comunicación y educación superior". En el Centro Universitario de Arte, Arquitectura y Diseño de la UdeG es miembro del Consejo de Centro, de la Junta Divisional, del Consejo Editorial y Secretario Ejecutivo del Comité de Compras y se ha desempeñado como Secretario Administrativo, Coordinadora de Investigación y Posgrado, Coordinadora de Personal y Jefa de la Unidad de Planeación. En la Coordinación de Cooperación Académica, UdeG, se desempeñó como Subcoordinadora Administrativa y Subcoordinadora de Cooperación Internacional. En el Centro de Estudios para Extranjeros, UdeG se desempeñó como Asistente de Promoción y Convenios.

\section{Mtro. Miguel Ángel Casillas López}

Maestro en Tecnologías de la Información por la Universidad de Guadalajara y profesor, desde 2009, de estructura de datos y plataformas operativas en el departamento de Sistemas de información, además de programación de sistemas por el Departamento de Ciencias Computacionales, Matemáticas para 
la Arquitectura en el Departamento de Técnicas y Construcción, y Gestión de la Infraestructura en TI de la Maestría en Tecnologías de la Información. Fue Jefe de la Unidad de Cómputo y telecomunicaciones durante tres años del Centro Universitario de Ciencias Económico Administrativas y actualmente Coordinador de Tecnologías para el Aprendizaje del Centro Universitario de Arte, arquitectura y Diseño. Ha realizado ponencias en el congreso de Innovación Tecnológica de Tecnologías de la Información, en el foro de actualización del plan de desarrollo Institucional de la Universidad de Guadalajara. Además es miembro del Consejo de Tecnologías de la Información, y miembro del comité para la innovación en Ambientes de Aprendizaje de la Universidad de Guadalajara.

\section{Mtro. Luis Alberto Mendoza Navarro}

Ingeniero en Comunicaciones y Electrónica y Maestro en Tecnologías para el Aprendizaje por la Universidad de Guadalajara. Docente del Centro Universitario de Arte, Arquitectura y Diseño de las materias de Computación I, Matemáticas, Calculo diferencial e integral y Matemáticas para Arquitectura. Actualmente es el secretario de la academia de Matemáticas. En el periodo 2008-2013 fue Jefe de la Unidad de Cómputo y Telecomunicaciones en el Centro Universitario de Ciencias Sociales y Humanidades. Actualmente es Jefe de la Unidad de Cómputo y Telecomunicaciones en el Centro Universitario de Arte, Arquitectura y Diseño. Realizó una ponencia en el foro de actualización del Plan de Desarrollo Institucional de la Universidad de Guadalajara. 